\title{
Evidence of Co-Circulation of Multiples Arbovirus Transmitted by Aedes Sp. Based on Laboratorial Syndromic Surveillance At Health Unit in Slum Area of Federal District, Brazil
}

\section{Paulo Rufalco-Moutinho ( $D$ paulorufalco@gmail.com )}

Universidade de Brasília Núcleo de Medicina Tropical: Universidade de Brasilia Nucleo de Medicina Tropical https://orcid.org/0000-0003-2540-2869

Lorena Aparecida Gonçalves de Noronha

Universidade de Brasilia

Tatyane de Souza Cardoso Quintão

Universidade de Brasilia Nucleo de Medicina Tropical

\section{Tayane Ferreira Nobre}

Universidade de Brasilia Nucleo de Medicina Tropical

\section{Ana Paula Sampaio Cardoso}

Universidade de Brasilia Nucleo de Medicina Tropical

\section{Daiani Cristina Cilião-Alves}

Universidade de Brasilia Nucleo de Medicina Tropical

Marco Aurélio Bellocchio Júnior

Universidade de Brasilia Nucleo de Medicina Tropical

Mateus de Paula von Glehn

Secretaria de Estado da Saude do Distrito Federal

\section{Rodrigo Haddad}

Universidade de Brasilia Nucleo de Medicina Tropical

\section{Gustavo Adolfo Sierra Romero}

Universidade de Brasilia Nucleo de Medicina Tropical

Wildo Navegantes de Araújo

Universidade de Brasília Núcleo de Medicina Tropical: Universidade de Brasilia Nucleo de Medicina Tropical

\section{Research Article}

Keywords: arboviruses, dengue, chikungunya, Aedes, syndromic surveillance, Brazil 
DOI: https://doi.org/10.21203/rs.3.rs-588530/v2

License: (c) (1) This work is licensed under a Creative Commons Attribution 4.0 International License. Read Full License 


\section{Abstract}

Background: Vector-borne diseases, especially arbiviruses transmitted by Aedes sp. mosquitos, should be a health policy priority in Brazil. Despite this urgency, there are significant limitations in the traditional surveillance system, such as lack of timely notification in identifying outbreaks at their onset and the systemic dismantling of entomological control in recent decades.

Methods: Laboratory syndromic surveillance for acute febrile and/or exanthematous syndromes was developed at a health unit in a favela (Portuguese for slum) of the Federal District in order to identify the circulation of arboviruses transmitted by Aedes sp. mosquitos. Between June/2019 and March/2020, 131 valid participants were identified and tested by RT-PCR for dengue (by serotype), Zika and Chikungunya virus acute infection; and ELISA-IgM for dengue fever and Chikungunya 15-21 days after symptom onset, when the participant reported no respiratory signs (cough and/or coryza). The results obtained were compared with traditional epidemiological surveillance data for the study area.

Results: At least 3 DENV-1 (2.3\%), 4 DENV-2 (3\%) and 1 CHIKV (0.7\%) cases were confirmed in the laboratory, showing evidence of hyperendemicity even though laboratory syndromic surveillance had not reached the historic peak dengue fever months in the Federal District (April-May). When the results obtained here were compared with traditional epidemiological surveillance data, a significant discrepancy was observed, including underreporting of Chikungunya infection.

Conclusions: in addition to the risks posed to the study population, the area investigated and its respective socioenvironmental profile may be a potential site for spreading the virus, given the cosmopolitan presence of Aedes sp. and human mobility in the Federal District. It is also suggested that traditional epidemiological surveillance may be notifying different acute viral infections such as dengue fever, while underreporting other arboviruses transmitted by Aedes sp. mosquitos in the Federal District.

\section{Background}

The emergence and reemergence of neglected infectious diseases (hitherto defined as tropical diseases) require continuous monitoring by the health agencies responsible for surveillance and intervention. This is especially important in Brazil, due to its continental size and large number of biomes under constant environmental disturbance, thereby promoting ecological imbalance. Among the main emerging infectious diseases, arboviruses transmitted by Aedes sp. mosquitos deserve the attention of health policies, as observed with the dengue virus in the Americas during the first two decades of the $21^{\text {st }}$ century. The continent had the largest number of notifications in the world, with Brazil contributing with 
the highest proportion and exhibiting an endemic-epidemic pattern every 3 to 5 years, in line with the prevalence of the serotype [1]. The country plays a key role in amplification and potentialization of mosquito-borne diseases, as stated by Bedin in 2007 [2], and demonstrated by the Aedes aegyptitransmitted Zika virus epidemic in 2015 which, despite its circulation in other countries, was only confirmed as having an association with congenital malformation syndrome after it emerged in Brazil [3].

With respect to the Aedes aegypti vector in Brazil, currently present in all the states of the country since its reemergence in the 1960s, the transmission of four dengue serotypes stand out: serotypes 1 and 4 in outbreaks during the 1980s, followed by serotype 2 in the 1990s, and serotype 3 in the 2000s [4]. It is also important to mention the Chikungunya virus, introduced in the country in 2013 [5], and the Zika virus in 2014 [3]. The species is also an urban vector for the yellow fever virus in Brazil, where repeated outbreaks were observed in recent years, associated with wild vectors (Hemagogus sp. and Sabethes sp.) in forest areas near urban zones [6]. Also important are other opportunistic arboviruses (mayaro, o'nyong-nyong, Oropouche, etc.), whose dynamics should be investigated [8,9] given the vector capacity of Aedes aegytpi [7]. Finally, Aedes albopictus, a secondary vector for the dengue virus in Brazil, is important in rural areas [10], but also observed in parks and green spaces in large cities [11].

This current hyperendemic scenario and the complex clinical findings associated with arbovirus infections, such as neuroinvasive and Guillian-Barré syndromes, systematically overload the Brazilian health system, affecting the country's therapeutic and economic capacities [12; 13]. In addition, the congenital malformation syndrome associated with Zika virus infection promotes this agent to the group of STORCH infections (syphilis, toxoplasmosis, rubella, cytomegalovirus, and herpes simplex), thereby requiring prenatal monitoring [14]. The sympatric circulation of different serotypes of dengue and other arboviruses promotes interactions in the physiopathogeny and immunological response of the human host, resulting in severe outcomes such as hemorrhagic shocks and death [15]. The consequence of new arboviruses with similar dynamics to that of the dengue virus produces more complex transmission, resulting in a broad symptomatological spectrum while decreasing the sensitivity and specificity of the clinical-epidemiological criterion for diagnosis, underscoring the need for laboratory confirmation [16]. The high number of assymptomatic and oligosyntomatic infections [17], as well as false-negative diagnoses due to the low sensitivity of widely used methods [18], mean outbreaks related to the arboviruses may occur without the epidemiological surveillance system's detecting them in a timely fashion, thereby facilitating their spreading and epidemics [19]. Also considered a complicating factor in controlling mosquito-borne diseases is the systematic dismantling of vector surveillance in recent decades in Brazil, which contrasts with the country's history of this strategy, such as the erradication of Aedes aegypti in the late 1950s [20].

In the present study, laboratory syndromic surveillance (LSS) was conducted for the three arboviruses transmitted by Aedes sp. mosquitos in a slum of the Federal District of Brazil, for an expected 1-year period, according to the seasonality of mosquito-borne diseases. This study was conducted in the only health unit of the region, assessing patients reporting symptoms associated with acute viral infection by the dengue (DENV), Chikungunya (CHIKV) and Zika viruses (ZIKV), following laboratory confirmation by 
molecular and serological tests. In Brazil, studies involving LSS for arboviruses transmitted by Aedes sp. were carried out in large coastal cities where the population is concentrated, such as the Northeast [12, $16]$ and Southeast regions [21,22], but not significantly replicated in the intra-continental portion $[23,24]$. The study area has previously been the object of epidemiological investigation based on cross-sectional interviews, reporting $28.6 \%$ of the symptoms associated with arbovirus infections in the sample population [25], albeit without laboratory confirmation. The present study aimed to determine the circulation of DENV, ZIKV and CHIKV in the study area; assess the clinical-epidemiological profile of laboratory confirmations; estimate the probable infection site for laboratory confirmations, identifying possible autochthonous transmissions, and; given that the study area is geographically isolated and the health unit is the only facility treating the vulnerable population, compare LSS results with traditional epidemiological surveillance data. It is important to underscore that the study concluded in March 2020, before the expected 1-year observation period (June 2020), due to the COVID-19 pandemic.

\section{Methods}

\section{Study Design}

Event-based LSS defined by acute arbovirus infection transmitted by Aedes sp. was conducted in a health unit, the only facility in the slum, and the first destination of individuals seeking medical care. Based on the definition of a probable case in the Manual for Adult and Child Diagnosis and Clinical Management for Dengue Fever [26], acute febrile and/or exanthematous illness was considered the defined event for LSS, thereby opting for broad spectrum symptomatology that addressed the symptomatic infections of the three arboviruses investigated here: for DENV, probable case symptomatology is defined as a sharp temperature increase $\left(39-40^{\circ} \mathrm{C}\right)$ accompanied by two or more of the following symptoms: headache, myalgia, arthralgia and retro-orbital pain, with exanthem present in $50 \%$ of symptomatic infections, as well as with anorexia, nausea, vomitting and diarrhea; for CHIKV, symptoms are similar, underscoring the intensity of arthralgia; for ZIKV, symptoms are characterized by absent or low to moderate-intensity fever, while exanthem occurs more frequently in the first days of the infection.

The sample population consisted of residents in the study area who visited the health unit in the morning (from 8:00 am to 12:00 pm) between Monday and Friday, complaining of acute fever and/or exanthemathous illness (the defined event). The primary identification of patients was conducted by family health teams (FHT) [27]. Each FHT is composed of a group of health professionals (doctor, nurse, community health agent) responsible for a geographic area within the respective region served by the health unit, which provides primary care. The health unit where LSS was carried out has $12 \mathrm{FHTs}$, which are instructed to refer patients presenting with the defined event. After the patient was referred, the following inclusion criteria were established: being older than 18 years of age; being a resident of the study area (at least four days a week for at least three months before the visit to the health unit); and not exhibiting any contraindication for venipuncture. The exclusion criteria were being a homeless person and illiterate (given the need to provide written informed consent). 
After the patients were referred by the FHT and had agreed to take part in the study, they signed the informed consent form and were given a brief explanation of the study objectives. Blood was collected characterized as the "acute moment" - and 15-21 days after symptom onset, participants were contacted again for a second blood collection - characterized as the "convalescent moment" - provided they did not exhibit cough and/or coryza (flu-like upper respiratory tract syndrome) [28]. The laboratory methodology recommended by the Ministry of Health [26] was applied for molecular and serological confirmation: RTPCR was used for the DENV (for serotypes), CHIKV and ZIKV in acute samples; and ELISA-IgM for DENV and CHIKV in both acute and convalescent samples. For the ELISA-IgM serological assay, two parameters were established: acute infection when seroconversion occurs, and recent infection when serology is positive in the acute sample [16]. To confirm acute infection, positive RT-PCR and/or seroconversion are considered laboratory evidence. ELISA-Igm was only tested for DENV due to the possible cross-reaction between dengue and Zika (both flaviviruses).

Based on the seasonal pattern of mosquito-transmitted diseases, an initial 1-year observation period was established for the present study, between June 2019 and June 2020. However, given the lack of knowledge regarding SARS-CoV-2 infection at the time, as well as the lack of biosecurity for conducting research at the health unit, it was decided to halt the study in March 2020, after 9 months of observation.

\section{Study Area}

LSS was conducted at Health Unit No. 4 ( $15^{\circ} 47$ '00.89 "S 47 $49^{\circ} 49.96^{\prime \prime}$ W) in the "Cidade Estrutural" administrative region (AR) of the Federal District (Figure 1-A). Cidade Estrutural is less than $10 \mathrm{~km}$ from the political center of Brazil and the executive, legislative and judiciary branches of government (Figure 1B). Established as a landfill site in the early 1960s, the area is one of the least developed ARs in the Federal District, with a vulnerable population that suffer from poverty and a lack of urban planning, infrastructure and environmental sanitation [29]. Albeit isolated, it is near the central region of the District: the Pilot Plan (plano piloto in Portuguese) (Figure 1-B). Cidade Estrutural is bordered by the Structural Highway to the south; the farm sector to the west; the "SCIA" industrial AR to the east; and the National Park of Brasilia to the north/northeast (Figure 1-C). Aproximately 35,730 inhabitants were estimated for 2018 , corresponding to $1.23 \%$ of the Federal District population of 2,894,953 [29]. According to the Federal District Health Department (Secretária de Saúde do Distrito Federal - SES-DF in Portuguese), the number of probable cases per 100,000 notified in the Cidade Estrutural in 2016, 2017, 2018 and 2019 was 1,$132 ; 475 ; 92.5$; and 1,613, respectively, and for the same period in the Federal District $583 ; 141 ; 79$; and 1,428 .

Figure 1. A: Location of the Federal District in Brazil; Federal District, highlighting Cidade Estrutural/SCIA in red, and Praça dos Três Poderes in yellow; B: Satellite image of the Pilot Project (central region of the Federal District) and surrounding area, highlighting Cidade Estrutural/SCIA in red and Praça dos Três Poderes in yellow; C: Satellite image of Cidade Estrutural, highlighting the key locations and homes with positive laboratory confirmations for the arboviruses investigated: $\mathrm{HU}$ : health unit; DD: deactivated 
dumping ground; NP: National Park of Brasilia; SCIA: AR bordering Cidade Estrutural, commercial and industrial area with a low number of houses. The homes with positive laboratory confirmations detected by LSS are represented by the following colors: Green: DENV-1; Red: DENV-2; Purple: CHIKV; Orange: DENV-IgM (seroconversion); Blue: recent DENV infection.

\section{Interview and Blood sampling}

Blood collection at the acute moment was performed using a tube with separator gel ( $5 \mathrm{~mL})$, followed by the interview to obtain demographic and socioeconomic characteristics (age, sex, ethnicity, address, domestic sanitary conditions); first day of symptoms; clinical characteristics of the event; daily routine in the last 15 days before symptom onset (school, university, work or travel); the epidemiological link to an individual (family, neighborhood, work or school mate) with a positive medical diagnosis for DENV. Between 15 and 21 days after symptom onset, participants who did not report cough and/or coryza were contacted for convalescent blood collection at the conclusion of the second part of the questionnaire (focusing on symptoms reported at the acute moment: cure or persistence).

A standardized questionnaire from the REDCap website, version 7.5.0 (www.project-redcap.org/) was applied using mobile devices (tablets).

\section{Laboratory Preparation}

Tubes containing the collected blood were kept at ambient temperature in the health unit laboratory, followed by centrifugation of $1500 \times \mathrm{g} / 10 \mathrm{~min}$, aliquoting the supernatant serum in triplicate microtubes (usually $0.5 \mathrm{~mL}$ in each microtube). Once duly labeled with the respective patient code, the samples were initially kept in the laboratory freezer of the health unit $\left(-20^{\circ} \mathrm{C}\right)$, then taken in thermal containers to the biorepository of the Center for Tropical Medicine, University of Brasilia (UnB) $\left(-80^{\circ} \mathrm{C}\right)$, where they were stored until molecular and serological analyses.

\section{Viral RNA extraction}

Viral RNA extraction of $140 \mu \mathrm{L}$ of serum from the acute sample was conducted using the QIAamp Mini kit, according to the manufacturer's recommendation (www.qiagen.com/us/resources), resulting in $60 \mu \mathrm{L}$ of eluate.

\section{Reverse transcription and arboviral RNA detection}

RT-PCR was performed using the "multiplex ZDC" kit from the Institute of Molecular Biology of Paraná with $38 \mu \mathrm{L}$ of eluate, in accordance with the manufacturer's recommendation (www.ibmp.org.br/en-us/). In this protocol [30], a standardized 96-well microplate is subdivided into four isometric subgroups of 24 wells for the respective target arboviruses: DENV 1/4; DENV 2/3; CHIKV; and ZIKV. Each of these four subgroups on the plate is composed of 23 wells to test the samples ( $38 \mathrm{uL} / 4$ subgroups $=9.5 \mathrm{uL}$ of eluate for each subgroup), and 1 positive control from the kit itself. RT-PCR was amplified using the QuantStudio 5 real-time system (http://thermofisher.com/), and the results analyzed by the company's software. 


\section{Serological testing for anti-DENV IgM}

Both acute and convalescent samples were tested for anti-DENV using the Panbio Dengue IgM Capture ELISA kit, according to the manufacturer's recommendation (www.globalpointofcare.abbott). Reading was done on a Kasuaki absorbance reader $(450 \mathrm{~nm})$, using Panbio units for cutoff values, following the criterion: $<9$ non-reactive; between 9 and 11 inconclusive; $>11$ reactive for anti-DENV IgM.

\section{Serological testing for anti-CHIKV IgM}

Both acute and convalescent samples were tested for anti-CHIKV using the Euroimun Chikungunya IgM ELISA kit, in line with the manufacturer's recommendation (www.euroimmun.com). Reading was done on a Kasuaki absorbance reader (450 nm), using relative units $(R U / m L)$ for cutoff values, based on the following criterion: $<0.8 \mathrm{RU} / \mathrm{mL}$ negative; between 0.8 and $1.1 \mathrm{RU} / \mathrm{mL}$ inconclusive; $>1.1 \mathrm{RU} / \mathrm{mL}$ positive for anti-CHIKV IgM.

\section{Data Analyses}

The number of defined events and laboratory confirmations was illustrated on epidemic curves, organized by epidemiological week (EW) starting with symptom onset. Clinical-epidemiological characteristics were cross-referenced with laboratory confirmations, using frequency tables. Information on routines for the 15 days prior to symptom onset was used to estimate the probable infection site.

In order to compare LSS results with traditional epidemiological surveillance data, probable arbovirus cases notified by the Cidade Estrutural and the Federal District were extracted from the Ministry of Health (datasus.gov.br) and Federal District Health Department websites (info.saude.df.gov.br): for the former, the interface allows extraction per period (year, month, EW) and final classification of the probable case (confirmed, discarded, not investigated), but not separation by the ARs of the Federal District; for the latter, the interface makes it possble to extract by period and site (including AR), but only dengue data are made available. Epidemiological bulletins from the Federal District Health Department were consulted to complement this information [31]. The final classification criteria of the probable case (clinicalepidemiological or laboratory) for Cidade Estrutural was obtained; the laboratory methods used (rapid NS1 test; IgM serology; PCR; viral isolation); and the dates of the first symptoms and blood collections for the respective laboratory tests established. Epidemic curves of probable dengue and Chikunguya cases from traditional surveillance for Cidade Estrutural were organized by EW starting on the day of symptom onset for the study period, discriminating the respective final classification (dengue, Chikunguya, discarded and non-investigated). Probable Zika cases and the respective final classification were not arranged by EW for the study area due to the non-availability of these notifications at the AR level for the Federal District. In order to observe the statistical differences between traditional surveillance and LSS results, the Wilcoxon test for paired data was conducted. To contextualize the COVID-19 situation in the first quarter of 2020, Federal District Health Department epidemiological bulletins from the Center of Emergency Operations for Covid-19, established in February of that year, were consulted [32]. 
Microsoft Excel was used to manage the datasets and create tables and graphs, Stata 14.0 software for statistical analyses, and Google Earth for satellite images. The GPS points of the dwellings were collected using a Garmin Etrex 10 handheld navigator.

\section{Results}

The study period was between the third week of June (EW 25) 2019 and third week of March (EW 12) 2020. A total of 157 individuals who presented with the defined event were referred by the FHT, 134 of whom were considered eligible for the study. The information on 3 of these was not saved electronically. At the end, 131 defined events were identified by the study. For the second blood collection (convalescent moment), the samples of 36 individuals were collected, given that 73 defined events exhibited cough and/or coryza; 15 individuals refused to participate, and 9 were not approached due to the early termination of the study.

Demographic and socioenvironmental characteristics are presented in Table 1. The LSS population samples consisted of adults aged around 30 years, with approximately 10 years of schooling, most of whom were non-white ( $89 \%)$ and women $(62.6 \%)$. Most of the population sample did not have private health insurance $(94.6 \%$ ) and approximately 4 of 5 individuals reported that their house has environmental sanitation (80\%), an indoor plumbing system (79\%) and trash collection (81\%). However, $40 \%$ declared having to ration water and $34.3 \%$ stored water by other methods in the three months before the interview.

Table 1. Demographic and socio-environmental characteristics of individuals identified by LSS at a Cidade Estrutural health unit, between June 2019 and March 2020. 


\begin{tabular}{cc}
\hline Variable & Values \\
\hline $\begin{array}{c}\text { Age, mean and SD } \\
\text { (years) }\end{array}$ & 32.2 and 12.8 \\
\hline $\begin{array}{c}\text { Age, median } \\
\text { (years) }\end{array}$ & 28 \\
$\begin{array}{c}\text { Sex } \\
\text { (female) }\end{array}$ & $62.6 \%$ \\
\hline $\begin{array}{c}\text { Ethnicity } \\
\text { (non-whites) }\end{array}$ & $89 \%$ \\
\hline $\begin{array}{c}\text { Years of schooling, mean and SD } \\
\text { (0, never attended; 16, university graduate) }\end{array}$ & 9.18 and 4,5 \\
\hline $\begin{array}{c}\text { Years of schooling, median } \\
\text { Y, never attended; 16, university graduate) }\end{array}$ & 10 \\
\hline \begin{tabular}{c} 
No health insurance \\
\hline Sewage collection at home
\end{tabular} & $94.6 \%$ \\
\hline Indoor plumbing & $80 \%$ \\
\hline $\begin{array}{c}\text { Water rationing } \\
\text { in the last 3 months }\end{array}$ & $30 \%$ \\
\hline $\begin{array}{c}\text { Stores water } \\
\text { by other methods }\end{array}$ & $34.3 \%$ \\
\hline Trash collection at home \\
\hline
\end{tabular}

Supplementary material 1 summarizes the flow of individuals from arriving at the health unit until laboratory confirmation. LSS identified 3 individuals with acute DENV-2 infection, testing positive for RTPCR and seroconversion; 1 individual with acute DENV-2 infection detected by RT-PCR, whose serologies at the convalescent moment were not performed; 3 individuals with acute DENV-1 infection (RT-PCR) whose serologies at the convalescent moment were also not performed (one of the participants refused the second collection; for the other two surveillance had already been terminated); 1 with acute DENV infection, detected only by seroconversion (DENV-IgM); 1 with acute CHIKV infection, detected only by seroconversion; and 3 recent DENV infections, as per a positive ELISA-IgM result at the acute moment and negative RT-PCR. The homes that exhibited positive laboratory confirmation were georeferenced (Figure 1-C).

Figure 2 shows three epidemic curves during the study period, adjusted according to collection time and the respective laboratory methodology used. The 4 confirmations of DENV-2 and 1 of DENV-IgM were observed between EW 25 and EW 29 (June-July) of 2019, while the 3 DENV-1 and 1 CHIKV confirmations were found between EW 4 and EW 12 (January-March) of 2020. No positive laboratory confirmation was observed in the period between these two clusters (second half of 2019). Despite the significant increase in defined events in the last EWs before the end of the study, only 1 positive laboratory confirmation was identified in the acute samples.

Figure 2. Epidemic curves for laboratory confirmations for LSS symptom onset data between EW 24 of 2019 and EW 12 of 2020, RT-PCR testing for acute samples (131); ELISA-IgM testing for acute samples 
(131); and ELISA-IgM testing for convalescent samples (36). A, B and C on the RT-PCR and Elisa-IgM curves for convalescent sample of the same individuals laboratory confirmed for acute DENV-2 infection.

The clinical-epidemiological characteristics of the 132 defined events and respective laboratory confirmations are shown in Table 2. Nearly all of the five most common symptoms associated with acute viral infections (headache, fever, myalgia, arthralgia and retro-orbital pain) were observed in positive laboratory confirmations, except for one DENV-1 without arthralgia and two DENV-2 without arthralgia and retro-orbial pain, respectively. Cough and coryza were observed in one DENV-1 and one DENV-2 case, respectively. Exanthem was present in DENV-IgM (identified in June 2019) and two DENV-1 confirmations (identified in January-March 2020). CHIKV stands out for the presence of sore throat. Although alarming signs were reported (difficulty breathing, bleeding), no severe dengue fever was found.

Table 2. Clinical-epidemiological characteristics and laboratory diagnosis of 131 defined events identified at the Cidade Estrutural health unit:

\section{Laboratory results}

\section{Clinical signs}

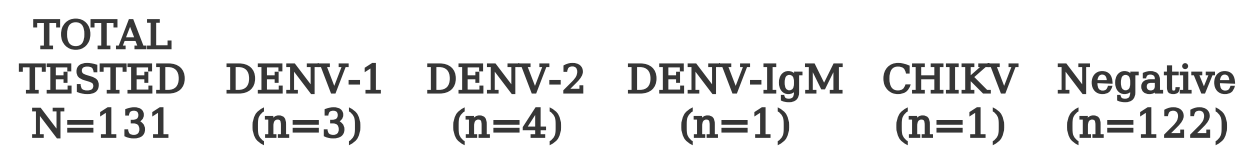

\begin{tabular}{rcccccc}
\hline Headache & 128 & 3 & 4 & 1 & 1 & 119 \\
\hline Fever & 125 & 3 & 4 & 1 & 1 & 116 \\
\hline Myalgia & 126 & 3 & 4 & 1 & 1 & 117 \\
\hline Arthralgia & 103 & 2 & 3 & 1 & 1 & 96 \\
\hline Retro-orbital pain & 102 & 3 & 3 & 1 & 1 & 94 \\
\hline Nausea & 92 & 1 & 3 & 0 & 1 & 87 \\
\hline Difficulty swallowing & 77 & 2 & 2 & 0 & 1 & 72 \\
\hline Shortness of breath & 55 & 0 & 2 & 0 & 1 & 53 \\
\hline Conjunctivitis & 55 & 2 & 1 & 1 & 0 & 51 \\
\hline Diarrhea & 44 & 1 & 2 & 0 & 0 & 41 \\
\hline Exanthem & 19 & 2 & 0 & 1 & 0 & 16 \\
\hline Vomiting & 35 & 0 & 2 & 0 & 0 & 33 \\
\hline Itching & 33 & 1 & 0 & 1 & 0 & 31 \\
\hline Cough & 66 & 1 & 0 & 0 & 0 & 65 \\
\hline Coryza & 62 & 0 & 1 & 0 & 0 & 61 \\
\hline Sore throat & 62 & 0 & 0 & 0 & 1 & 61 \\
\hline ding (nose, mouth, feces) & 18 & 2 & 0 & 0 & 0 & 16 \\
\hline Oral mucosal lesion & 15 & 1 & 0 & 0 & 0 & 14 \\
\hline
\end{tabular}


The main characteristics related to the daily routines and possible exposure for laboratory confirmations by LSS are presented in Supplementary Material 2. Individuals from samples 39 (DENV-2), 98 (CHIKV) and 106 (DENV-1) were not exposed outside their homes, and it is plausible to characterize these cases as household transmission. For individual 27 (DENV-IgM), Cidade Estrutural can also be considered the probable infection site (residence or workplace). The workplace of individual 37 (DENV-2) is in the neighboring AR (SCIA, see Figure 2-C), but since this individul works only at night, when Aedes aegypti activity declines significantly [33], household transmission is also suggested in this instance.

Figure 3 shows five epidemic curves: the first two, from top to bottom, corresponding to traditional surveillance notifications of probable dengue cases for the Federal District and Cidade Estrutural, respectively, between EW 1 of 2019 and EW 25 of 2020. The three remaining Cidade Estrutural curves during the study (EW 25 of 2019 to EW 12 of 2020), respectively, are for probable dengue cases notified (characterized according to the final classification); probable Chikungunya cases notified (characterized according to the final classification); and LSS results. The traditional surveillance data for the FD show that significant peaks of probable dengue cases occurred during the first half of both years (2019 and 2020), but only in 2019 was a peak observed in Cidade Estrutural. There is a pandemic bias for 2020, contextualized in the Discussion.

Figure 3. From top to bottom, epidemic curves of symptom onset for traditional surveillance of probable dengue cases in the Federal District between EW 1 of 2019 and EW 25 of 2020; traditional surveillance of probable dengue cases in Cidade Estrutural betwen EW 1 of 2019 and EW 25 of 2020; traditional surveillance of probable dengue cases and final classification for Cidade Estrutural between EW 25 of 2019 and EW 12 of 2020; traditional surveillance of probable Chikungunya cases and final classification for Cidade Estrutural between EW 25 of 2019 and EW 12 of 2020; and LSS results between EW 25 of 2019 and EW 12 of 2020.

Figure 3 shows the comparisons between traditional surveillance notifications (174 probable dengue cases during the study period, 94 classified as dengue, 70 discarded and 10 not investigated; and 5 probable Chikungunya cases, all classified as dengue) and LSS results (126 defined events, with 9 laboratory confirmations of $\mathrm{CHIKV}$ ), revealing differences between magnitudes and distributions. In regard to the EWs for LSS confirmations, the first cluster exhibited 5 confirmations of acute infection (4 DENV-2 and 1 DENV-IgM) between SE 25 and SE 29 of 2019, the same period traditional surveillance notified 23 dengue cases; and the second cluster 4 confirmations of acute infection (3 DENV-1 and 1 CHIKV) between SE 4 and 12 of 2020, the same period in which traditional surveillance notified 45 dengue cases. Information on traditional surveillance also indicates dengue cases being notified during the second half of 2019, a pattern not observed by LSS. Although the probable Chikungunya cases of traditional surveillance are temporally close to LSS confirmation, they were classified as dengue.

With respect to statistical analyses between traditional surveillance data and LSS results, considering a $95 \% \mathrm{Cl}$ and the null hypothesis that the magnitudes of SE-paired values are equal, differences were observed for all the comparisons performed: between probable dengue cases and defined events 
( $p=0.0346)$; between dengue cases and defined events ( $p=0.0238)$; and between dengue cases and laboratory confirmations $(p=0.000)$. In terms of the traditional surveillance criteria used to confirm probable dengue cases for Cidade Estrutural in 2019, 571 of the 652 probable cases notified were thoroughly investigated, 216 using clinical-epidemiological criteria and 355 laboratory methods. However, these numbers may be inconsistent, since 360 rapid tests were conducted for NS1, in addition to 167 serological IgM, 5 PCR and 1 viral isolation test. For 2020, 310 of the 312 probable dengue cases notified were thoroughly investigated: 44 using clinical-epidemiological criteria and 266 laboratory methods (231 by rapid NS1 testing, 157 by IgM serology, 24 by PCR and 1 by viral isolation).

\section{Discussion}

Sentinel units are an important strategy for data collection in health surveillance, and are selected based on the probable occurrence of outcomes of interest, with the area receiving intensive monitoring from the specialized team [34]. For epidemiological surveillance, hospitals and outpatient facilities are important sentinel units for diseases and complications related to human health, since these establishments are the entry points to the health system. In relation to infectious diseases, the natural history and transmission pathway should be parameters for selecting sentinel units. Vulnerable areas such as Brazilian favelas are hot spots for mosquito-transmitted diseases, according to the association between Aedes sp. biology and regions with and without environmental sanitation [16]. Health unit N04 in Cidade Estrutural is an important satellite for epidemiological surveillance in the Federal District for investigating the vulnerable population of the Pilot Plan, a situation that underscores the extreme inequality that still exists in Brazil. Given the advantages of well-incorporated and consolidated syndromic surveillance [35], producing accurate real-time results for health authorities [36], opportunities for intervention and control are created before pathogen dissemination. For mosquito-borne diseases, entomological surveillance should also be incorporated to block the transmission chains. These different approaches, which naturally complement each other, represent integrated surveillance [37], an important ally in reducing the number of vector-borne diseases, and may even culminate in erradication [38;39].

The study found evidence of two DENV serotypes and CHIKV circulating, with high plausibility of at least three individuals having been infected in their homes (positive for DENV-1, DENV-2 and CHIKV). Cidade Estrutural is hyperendemic for arboviruses transmitted by Aedes sp. mosquitos, with the population exposed to the risks of secondary infection, co-infection and clinical outcomes in areas where this "arbovirus soup" occurs [15], despite cross-immunity being observed among flavivirus infections [40;41]. The site is a potential area for the spreading of these arboviruses to other ARs, given the cosmopolitan presence of Aedes aegypti throughout the Federal District and the human mobility that occurs in the region.

The possible low number of laboratory confirmations by LSS for the population sample may be due to the inclusion of nonspecific symptoms in the clinical spectrum of the defined event, including upper respiratory tract symptoms (cough 66; coryza: 62; both 53) which are not associated with the definition of probable cases for arboviruses transmitted by Aedes sp. [26]. This option was adopted to maximize 
sampling for detection by laboratory methodologies, and to observe the clinical profile of laboratory confirmations. However, when compared to other LSS studies on arboviruses transmitted by Aedes sp. conducted in health units in the country, the number of positive DENV cases observed here (6.1\%) was higher than that reported by Silva et al. 2019 (3.4\%) [16], Ferreira et al. $2020(0.4 \%)$ [12], Carvalho et al. $2020(0.8 \%)$ [21] and Vieira et al. $2019(n=0)$ [24]. For the other two arboviruses, the other studies obtained higher results. In addition to the particularities of each investigation and the study population, this discrepancy depends on whether outbreaks and epidemics occur while LSS is being carried out, as occurred in the study by Silva et al. 2019 [16] during the 2015 Zika epidemic in Salvador, Bahia state.

Laboratory confirmations exhibited the five most common symptoms involving acute viral infections, confirming the low specificity for diagnosing arbovirus infection when not supported by laboratory analysis [16]. Cough and coryza were observed in 1 DENV-1 and 1 DENV-2 confirmation, respectively. This aspect of acute viral syndromes is used to discriminate airborne viruses from others (such as arboviruses) [28]. However, albeit uncommon, upper respiratory tract symptoms may be associated with acute arboviral infections transmitted by Aedes sp., including cough, coryza and nasal congestion [42]. It is important to underscore that DENV has been isolated in secretion samples collected from the upper respiratory tract, raising the hypotheis of possible transmission via aerossol or close contact, as observed in the replication of this virus in culture mediums of airway epithelium cells. However, evidence is still insufficient to confirm this infection pathway [43].

Given the acute DENV infection confirmed only for seroconversion (DENV-IgM), the possible crossreaction between flaviviruses in immunoenzymatic assays for $\lg \mathrm{M}$, and that exanthem is associated with acute ZIKV infection, suggest the transmission of this virus. The low viral load observed for ZIKV in blood serum, when compared to DENV, [44] reinforces the suggestion of infection by the former (which also explains the more intense fever in DENV than in ZIKV infection). For ZIKV, testing in other biological specimens such as urine was recommended (especially for pregnant women suspected of being infected) [45]. Although this seroconversion has been identified in the DENV-2 cluster (between EW 25 and 29 of 2019), suggesting infection of this dengue virus serotype, none of these DENV-2 exhibited enxanthem, only confirmations for DENV-1 located in the other cluster (between EW 4 and EW 12 of 2020). The low ZIKV prevalence in the Federal District during the study period reinforces seroconversion to DENV-2 (2019: confirmaded $=66$; discarded= 198; inconclusive $=135 ; 2020$ : confirmed $=10$; discarded= 2267; inconclusive=36). However, other arboviruses transmitted by Aedes sp. may be underreported (discussed in the next paragraphs).

Traditional surveillance notifications show that the difference between probable dengue cases in the Federal District and Cidade Estrutural indicates that the latter did not contribute to the total in 2020, only 2019. The COVID-19 pandemic represents a potential bias for probable case notifications for 2020 , starting in March (Supplementary Material 3). Comparisons between traditional surveillance notifications and LSS results show important discrepancies, given that it is an isolated area with a vulnerable population dependent on the health unit. Moreover, because traditional surveillance such as LSS had the same source of patients in the health unit, some similarity was expected between the values of the two 
surveillance methods. However, limitations included the communication between LSS and a number of FHTs; LSS was performed only in the morning (8:00 am-12:00 pm); the more encompassing symptomatological spectrum of the defined event of LSS when compared to the definition of probable arbovirus cases; only individuals aged 18 years or older participated in the study; and possible notification sources other than the health unit may explain the differences between the numbers of both surveillance methods.

The obvious contrast between dengue cases notified by traditional surveillance and LSS confirmations indicates that different acute viral infections may be notified as dengue. The absence of confirmed notification of Chikungunya, with probable cases of this virus classified as dengue, suggests underreporting. The confirmation criteria of probable cases reported by traditional surveillance for the study area and the laboratory methodologies used demonstrate the inconsistencies for 2019 [46], namely that the laboratory criteria value (355) was lower than that of rapid NS1 tests (360). With respect to laboratory criteria sensitivity, it is known that the rapid tests routinely used for point-of-care in outpatient facilities may exhibit low sensitivity when compared to those obtained in the laboratory [47]. For IgM serology, the measures of central tendency for the set of values obtained by the difference between the day of symptom onset and the blood collection day for $2019(n=167)$ show a mean of 10.4 days; SD of 6.4 days; median of 3 days; and mode of 2 days; and for $2020(n=157)$ mean of 4.3 days; SD of 6.6 days; median of 3 days; and mode of 3 days. These indicators reveal that a large number of samples were collected within 1 week of symptom onset, suggesting that recent infections can be classified as acute, according to IgM circulation time in the bloodstream (3 months or more after the end of the infection) [26]. Depending on the seasonality of mosquito-borne diseases, longer transmissibility times may maximize this bias. PCR and viral isolation were less frequently used than the rapid NS1 tests and IgM serology. However, for PCR, there was an increase between 2019 (5 PCRs/652 probable dengue cases = 0.0007 PCR per probable case) and 2020 (24 PCRs $/ 312$ probable dengue cases $=0.077$ PCR per probable case), probably influenced by the COVID-19 pandemic in 2020 .

Given the limitations of the clinical-epidemiological criterion, and dependence on an effective flow for laboratory confirmation of infectious diseases, it can be suggested that different acute infections such as DENV are notified for the study area, while other arboviruses are underreported. Several areas of Brazil have a vulnerable population that are dependent on the National Health System (SUS, in Portuguese), which has undergone a structural and financial decline in recent years [48]. The worst scenario has been observed during the COVID-19 pandemic, considering the areas with active arbovirus transmission [49]. The subsequent endemic circulation of SARS-CoV-2 in the Brazilian population will be a further hindrance to the notification of acute viral syndromes when not confirmed by laboratory criteria.

A number of limitations were observed in the Cidade Estrutural health unit. First, communication with different FHTs produced heterogeneous identification of defined events during the study period, due to limitations of the teams themselves. Second, the LSS could not be maintained during the daily operation of the health unit (8:00 am-6:00pm), and not collecting the convalescent sample of some patients contributed to reducing the sampling effort for laboratory analysis. Third, the COVID-19 pandemic 
precluded concluding the 1-year observation period and delayed the laboratory processing of biological samples, since resources and personnel were redirected to combat the new virus. The pandemic can also be considered a potential bias for the FHT in identifying defined events and traditional surveillance data in 2020. However, Supplementary Material 3 and the epidemiological bulletin for COVID-19 [32] reported the first confirmed notification on March 5 for an individual who had traveled to Europe; the second confirmation occurred on March 10 (wife of the first case); 19 confirmations on March 16; and 87 on March 20, 5 of which classified as local transmission, meaning that January and the first three weeks of February (SE1 to SE7) can still be considered pre-pandemic period. This is also supported by the Ministry

of Health's recognizing the existence of community transmission of SARS-CoV-2 on March 20 [32]. In this pre-pandemic context, LSS identified DENV-2 (SE 4) and CHIKV (SE 7).

LSS results raise questions regarding the time elapsed between laboratory confirmations of DENV-2 and DENV-1 during the second half of 2019, and the absence of confirmations between these two clusters. Are these transmissions sustained at the local level in the study area during low Aedes sp. density, depending on the seasonality of Aedes aegypti-borne diseases? In addition, does any type of ecological substitution of DENV serotypes occur during this seasonality? Thus, to answer these questions, active surveillance should be performed (also focusing on asymptomatic individuals), along with entomological surveillance and analyses of mosquito infectivity.

\section{Conclusion}

Despite the limitations, LSS identified autochthonous household transmission for two different DENV and CHIKV serotypes. When these results are compared with those of traditional surveillance, there is a significant discrepancy, suggesting that other acute viral syndromes be classified as dengue, and that other arboviruses transmitted by Aedes sp. are underreported. Given the high number of negative laboratory results observed by LSS, acute syndromes caused by other viruses can be speculated, including airborne virus, as demonstrated by upper respiratory tract symptoms (flu-like syndrome), which circulated in the Federal District before the COVID-19 pandemic (influenza virus; adenovirus; rhinovirus; earlier coronaviruses; etc.), indicating the importance of continuous LSS for surveillance strategies. Integrated surveillance (traditional, sisyndromic, laboratory-based, genomic, entomological), in addition to hybrid systems and new data science methodologies [50], are essential instruments in predicting the emergence of new and reemergence of earlier infectious agents in this brave new world.

\section{List Of Abbreviations}

STORCH: syphilis, toxoplasmosis, rubella, cytomegalovirus, and herpes simplex

LSS: laboratory syndromic surveillance

DENV: dengue virus

ZIKV: Zika virus 
CHIKV: chikungunya virus

FHT: Family Health Team

AR: Administrative Region of Federal District, Brazil

FD: Federal District

EW: epidemiologic week

\section{Declarations}

\section{Ethics approval and consent to participate}

Valid participants were informed about the project's objectives and respective proceedings, as well as the risks and benefits involved. Those who agreed to participate voluntarily signed an Informed Consent Form. The research complied the resolution 466/2012 of the National Health Council and the principles of the Declaration of Helsinki, and was approved by the Ethics Committees in Research involving human beings from University of Brasília (CAAE: 61551116.3.3001.5558); and from State Health Secretariat of the Federal District (CAAE: 61551116.3.0000.5553).

\section{Consent for publication}

Not applicable.

\section{Availability of data and materials}

The datasets used and/or analyzed during the current study are available from the corresponding author on reasonable request.

\section{Competing interests}

The authors declared there are no competing interests in the development of the research.

\section{Funding}

This research was partially supported by Fundação de Apoio à Pesquisa do Distrito Federal (FAPDF) Edital 04/2016 n 0193.000.416/2016, and by grant “Prevenção e Combate ao vírus Zika" by Conselho Nacional de Desenvolvimento Científico e Tecnológico (CNPq) n 440844/2016-9 and Coordenação de Aperfeiçoamento de Pessoal de Nível Superior (CAPES) nº 88881.130785/2016-01.

\section{Authors' contributions}

PRM, MABJ, MPG, RH and WNA conceptualized the study; PRM, MABJ and MPG performed investigation and field collection; PRM performed data curation; PRM, LAGN, TSCQ, TFN, APSC performed laboratorial 
methodology; PRM, LAGN, TSCQ, TFN, DCCA and RH performed laboratorial analysis; PRM interpreted the results, wrote the original draft of manuscript and designed the visualization; PRM, TSCQ, TFN, RH, GASR and WNA reviewed and edited the final manuscript. All authors read and approved the final manuscript.

\section{Acknowledgements}

Ana Cláudia Negret Scalia (NMT/UnB); Elias Luiz Batista Cavalcante (FCe/UnB); members from the ZARICS Research Team and professionals from NMT/UnB; the health professionals from the Health Unit N. ${ }^{\circ} 4$; and the residents of Cidade Estrutural for the hospitality.

\section{References}

1. Salles TS, da Encarnação Sá-Guimarães T, de Alvarenga ESL, Guimarães-Ribeiro V, de Meneses MDF, de Castro-Salles PF, et al. History, epidemiology and diagnostics of dengue in the American and Brazilian contexts: a review. Parasit Vectors. 2018; 11(1):264.

2. Bedin F. Brazil, land of choice for arbovirosis?. Med Trop (Mars); 2007 67(3):281-7.

3. Teixeira MG, Costa Mda C, de Oliveira WK, Nunes ML, Rodrigues LC. The Epidemic of Zika VirusRelated Microcephaly in Brazil: Detection, Control, Etiology, and Future Scenarios. Am J Public Health. 2016;106(4):601-605

4. Nunes, PCG, Daumas RP, Sánchez-Arcila JC, Nogueira RMR, Horta MAPH, Santos FB. 30 years of fatal dengue cases in Brazil: a review. BMC Public Health. 2019; 19: 329.

5. Souza TMA, Ribeiro ED, Corrêa VCE, Damasco PV, Santos CC, de Bruycker-Nogueira F, et al. Following in the Footsteps of the Chikungunya Virus in Brazil: The First Autochthonous Cases in Amapá in 2014 and Its Emergence in Rio de Janeiro during 2016. Viruses. 2018; 12;10(11):623.

6. Giovanetti M, de Mendonça MCL, Fonseca V, Mares-Guia MA, Fabri A, Xavier J, et al. Yellow Fever Virus Reemergence and Spread in Southeast Brazil, 2016-2019. J Virol. 2019; 12;94(1):e01623-19.

7. Carvalho FD, Moreira LA. Why is Aedes aegypti Linnaeus so Successful as a Species? Neotropical Entomology. 2017, 46: 243-255.

8. Pezzi L, Diallo M, Rosa-Freitas MG, Vega-Rua A, Ng LFP, Boyer S, et al. GloPID-R report on chikungunya, o'nyong-nyong and Mayaro virus, part 5: Entomological aspects. Antiviral Res. 2020, Feb;174:104670.

9. Romero-Alvarez D, Escobar LE. Oropouche fever, an emergent disease from the Americas. Microbes Infect. 2018, 20(3):135-146.

10. Pancetti et al., 2015, Twenty-eight years of Aedes albopictus in Brazil: a rationale to maintain active entomological and epidemiological surveillance. Rev Soc Bras Med Trop . Jan-Feb 2015;48(1):87-9.

11. Heinisch et al., 2019, Seasonal and spatial distribution of Aedes aegypti and Aedes albopictus in a municipal urban park in São Paulo, SP, Brazil. Acta Trop, 189:104-113.

12. Ferreira MLB, Albuquerque MFPM, Brito CAA, FRança RFO, Moreira AJP, Machado MIM. Neurological disease in adults with Zika and chikungunya virus infection in Northeast Brazil: a prospective 
observational study. The Lancet Neurology. 2020, 19(10): 826-839.

13. Peixoto, HM, Romero, GAS, Araújo, WN, Oliveira, MRF. Guillain-barré syndrome associated with zika virus infection in brazil: a cost-of-illness study. Transactions of the royal society of tropical medicine and hygiene. 2019, 113: 252-258.

14. Gallo LG, Martinez-Cajas J, Peixoto HM, Pereira ACES, Carter JE, McKeown, et al. Another piece of the Zika puzzle: assessing the associated factors to microcephaly in a systematic review and metaanalysis. BMC Public Health. 2020, 20: 827.

15. Paixão ES, Teixeira MG, Rodrigues LC. Zika, chikungunya and dengue: the causes and threats of new and re-emerging arboviral diseases. BMJ Global Health. 2018, 4;3(Suppl 1):e000530.

16. Silva MMO, Tauro LB, Kikuti M, Anjos RO, Santos VC, Gonçalves TSF, et al. Concomitant Transmission of Dengue, Chikungunya, and Zika Viruses in Brazil: Clinical and Epidemiological Findings From Surveillance for Acute Febrile Illness. Clin Infect Dis. 2019, 27;69(8):1353-1359.

17. Díaz-Quijano FA, Waldman EA. Factors Associated with Dengue Mortality in Latin America and the Caribbean, 1995-2009: An Ecological Study. 2012. Am. J. Trop. Med. Hyg., 86(2): 328-334.

18. Jääskeläinen AJ, Korhonen EM, Huhtamo E, Lappalainen M, Vapalahti O, Kallio-Kokko H. Validation of serological and molecular methods for diagnosis of zika virus infections. J Virol Methods. 2019, 263:68-74.

19. Rojas DP, Dean NE, Yang Y, Kenah E, Quintero J, Tomasi S, et al. The epidemiology and transmissibility of Zika virus in Girardot and San Andres island, Colombia, September 2015 to January 2016. Euro Surveill. 2016, 14;21(28):10.2807.

20. Araújo HR, Carvalho DO, loshino RS, Costa-da-Silva AL, Capurro ML. Aedes aegypti Control Strategies in Brazil: Incorporation of New Technologies to Overcome the Persistence of Dengue Epidemics. Insects. 2015;6(2):576-594. Published 2015 Jun 11. doi:10.3390/insects6020576

21. Carvalho FR, Medeiros T, Vianna RAO, Douglass-Jaimes G, Nunes PCG, Quintans MDS, et al. Simultaneous circulation of arboviruses and other congenital infections in pregnant women in Rio de Janeiro, Brazil. Acta Trop. 2019, 192:49-54.

22. Estofolete CF, Terzian ACB, Colombo TE, de Freitas Guimarães G, Ferraz HC Junior, da Silva RA, et al. Co-infection between Zika and different Dengue serotypes during DENV outbreak in Brazil. J Infect Public Health. 2019, 12(2):178-181.

23. Souza Costa MC, Siqueira Maia LM, Costa de Souza V, Gonzaga AM, Correa de Azevedo V, Ramos Martins $L$, et al. Arbovirus investigation in patients from Mato Grosso during Zika and Chikungunya virus introdution in Brazil, 2015-2016. Acta Trop. 2019, 190:395-402.

24. Vieira DS, Zambenederri MR, Requião L, Borghetti IA, Luna LKS, Santos AO. Epidemiological profile of Zika, Dengue and Chikungunya virus infections identified by medical and molecular evaluations in Rondonia, Brazil. Rev Inst Med Trop Sao Paulo. 2019, 61:e40.

25. Cruvinel VRN, Marques CP, Cardoso V, Novaes MRCG, Araújo WN, Angulo-Tuesta A. Health conditions and occupational risks in a novel group: waste pickers in the largest open garbage dump in Latin America. BMC Public Health. 2019, 19:581. 
26. Ministério da Saúde do Brasil: Dengue, diagnóstico e manejo clínico adulto e criança, 4a edição, Brasília-DF, 2016

27. Costa NR. The Family Health Strategy: primary health care and the challenge of Brazilian metropolises. Ciênc. saúde colet. 2016, 21 (5): 1389-1398.

28. Cavailler, P., Tarantola, A., Leo, Y.S. et al. Early diagnosis of dengue disease severity in a resourcelimited Asian country. BMC Infect Dis 16, 512 (2016).

29. PDAD - Pesquisa Distrital por Amostra de Domicílios, SCIA/Estrutural. Brasilia-DF,2018 http://www.codeplan.df.gov.br/wpcontent/uploads/2020/06/SCIA-Estrutural.pdf.

30. Instituto de Biologia Molecular do Paraná, KIT BIOMOL ZDC http://www.ibmp.org.br/pt-br/wpcontent/uploads/2020/05/Instru\%C3\%A7\%C3\%A3o-de-Uso-Kit-BIOMOL-ZDC-rev-03.pdf

31. Secretaria de Saúde do Distrito Federal, Informativos Epidemiológicos Dengue, Chikungunya, Zika e Febre Amarela https://www.saude.df.gov.br/informes-dengue-chikungunya-zika-febre-amarela/

32. Secretaria de Saúde do Distrito Federal, Boletins Informativos sobre a Covid-19 no Distrito Federal (Divep/SVS) https://www.saude.df.gov.br/boletinsinformativos-divep-cieves/

33. Carvalho FD, Moreira LA. Why is Aedes aegypti Linnaeus so Successful as a Species?. Neotrop Entomol. 2017, 46:243-255.

34. Teixeira Md Mda G, Barreto ML, Costa Mda C, Strina A, Martins D Jr, Prado M. Sentinel areas: a monitoring strategy in public health. Cad Saude Publica. 2002 Sep-Oct;18(5):1189-95. doi: 10.1590/s0102-311x2002000500011. PMID: 12244351.

35. Henning KJ. What is Syndromic Surveillance? Morbidity and Mortality Weekly Report. 2004, 53(Suppl):5-11

36. Brasil. Ministério da Saúde. Secretaria de Vigilância em Saúde. Departamento de Vigilância Epidemiológica. Guia de vigilância epidemiológica / Ministério da Saúde, Secretaria de Vigilância em Saúde, Departamento de Vigilância Epidemiológica. - 7. ed. - Brasília: Ministério da Saúde, 2009.

37. Guzman MG, Halstead SB, Artsob H, Buchy P, Farrar J, Gubler DJ. Dengue: a continuing global threat. Nat Rev Microbiol. 2010, 8(12S):7-16.

38. Perera WDHN, Gunathilaka PADHN, Taylor-Robinson AW. Malaria in Sri Lanka: Investigating causes of the recent elimination and making plans to prevent reintroduction, J Vector Borne Dis. 2019, 56(3):179-188.

39. Zhang S et al., 2018, Malaria Elimination in the People's Republic of China: Current Progress, Challenges, and Prospects. Towards Malaria Elimination: A Leap Forward. Chapter 10

40. Damasceno L, Terzian ACB, Fuller T, Estofolete CF, Andrade A, Kroon EG, Zin AA, Vasconcelos Z, Pereira JP Jr, Castilho MC, Piaulino ICR, Vasilakis N, Moreira ME, Nielsen-Saines K, Espinosa FEM, Nogueira ML, Brasil P. Why Did ZIKV Perinatal Outcomes Differ in Distinct Regions of Brazil? An Exploratory Study of Two Cohorts. Viruses. 2021, 13(5):736.

41. Pedroso C, Fischer C, Feldmann M, Sarno M, Luz E, Moreira-Soto A, Cabral R, Netto EM, Brites C, Kümmerer BM, Drexler JF. Cross-Protection of Dengue Virus Infection against Congenital Zika 
Syndrome, Northeastern Brazil. Emerg Infect Dis. 2019, (8):1485-1493.

42. Gregory CJ, Santiago LM, Argüello DF, Hunsperger E, Tomashek KM. Clinical and Laboratory Features That Differentiate Dengue from Other Febrile Illnesses in an Endemic Area-Puerto Rico, 2007-2008. The American Journal of Tropical Medicine and Hygiene. 2010;82(5):922-9.

43. Cheng NM, Sy CL, Chen BC, Huang TS, Lee SS, Chen YS. Isolation of dengue virus from the upper respiratory tract of four patients with dengue fever. PLoS Negl Trop Dis. 2017, 5;11(4):e0005520.

44. Musso D, Rouault E, Teissier A, Lanteri MC, Zisou K, Broult J, Grange E, Nhan TX, Aubry M. Molecular detection of Zika virus in blood and RNA load determination during the French Polynesian outbreak. J Med Virol. 2017 Sep;89(9):1505-1510. doi: 10.1002/jmv.24735. Epub 2017 May 23. PMID: 27859375; PMCID: PMC5575554.

45. Sanchez Clemente N, Brickley EB, Furquim de Almeida M, Witkin SS, Duarte Passos S, Jundiai Zika Cohort Group T. Can Zika Virus Infection in High Risk Pregnant Women Be Differentiated on the Basis of Symptoms? Viruses. 2020 Nov 5;12(11):1263. doi: 10.3390/v12111263. PMID: 33167566; PMCID: PMC7694531.

46. MMWR Recommendations and Reports: Past Volume, July 27, 2001 / Vol. 50 / No. RR-13 / Pg. 1 36

47. Kikuti M, Cruz JS, Rodrigues MS, Tavares AS, Paploski IAD, Silva MMO, Santana PM, Tauro LB, Silva GAOF, Campos GS, Araújo JMG, Kitron U, Reis MG, Ribeiro GS. Accuracy of the SD BIOLINE Dengue Duo for rapid point-of-care diagnosis of dengue. PLoS One. 2019 Mar 6;14(3):e0213301. doi: 10.1371/journal.pone.0213301. PMID: 30840708; PMCID: PMC6402663.

48. Demo MLO, Orth LC, Marcon CEM. Brazil's health-care system. The Lancet Correspondence. 2019; 394(10213):1992

49. Silva SJRD, Magalhães JJF, Pena L. Simultaneous Circulation of DENV, CHIKV, ZIKV and SARS-CoV-2 in Brazil: an Inconvenient Truth. One Health. 2020, 16;12:100205.

50. Simonsen L, Gog JR, Olson D, Viboud C. Infectious Disease Surveillance in the Big Data Era: Towards Faster and Locally Relevant Systems. J Infect Dis. 2016; 214(suppl_4):S380-S385.

\section{Figures}





\section{Figure 1}

A: Location of the Federal District in Brazil; Federal District, highlighting Cidade Estrutural/SCIA in red, and Praça dos Três Poderes in yellow; B: Satellite image of the Pilot Project (central region of the Federal District) and surrounding area, highlighting Cidade Estrutural/SCIA in red and Praça dos Três Poderes in yellow; C: Satellite image of Cidade Estrutural, highlighting the key locations and homes with positive laboratory confirmations for the arboviruses investigated: HU: health unit; DD: deactivated dumping 
ground; NP: National Park of Brasilia; SCIA: AR bordering Cidade Estrutural, commercial and industrial area with a low number of houses. The homes with positive laboratory confirmations detected by LSS are represented by the following colors: Green: DENV-1; Red: DENV-2; Purple: CHIKV; Orange: DENV-IgM (seroconversion); Blue: recent DENV infection.

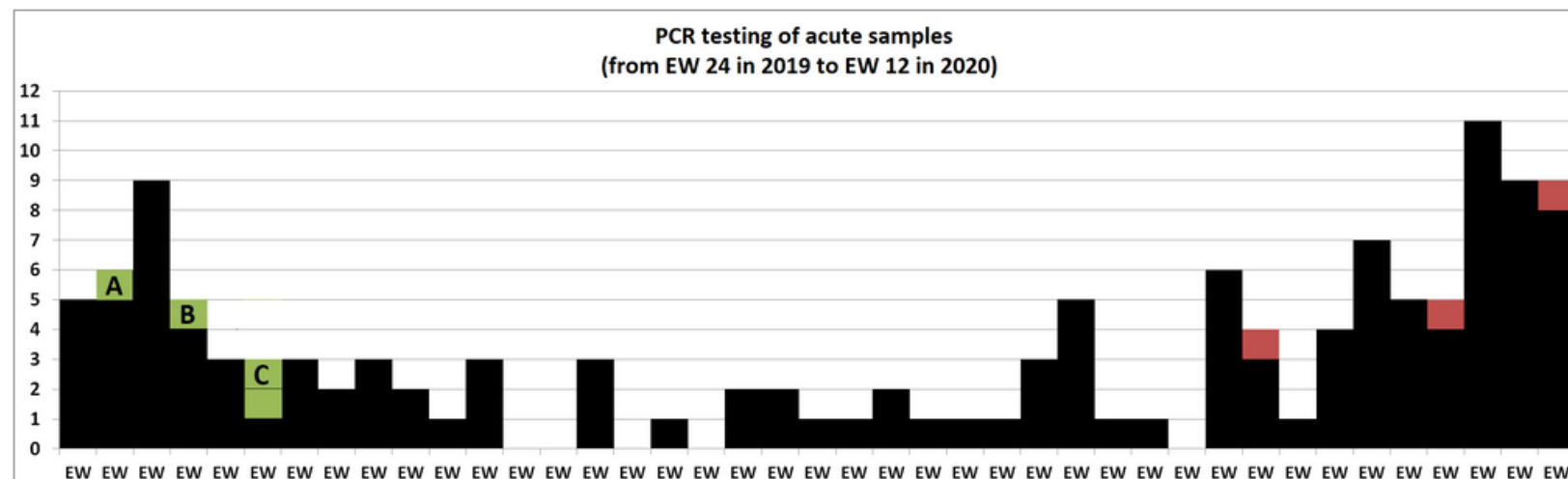

EW EW EW EW EW EW EW EW EW EW EW EW EW EW EW EW EW EW EW EW EW EW EW EW EW EW EW EW EW EW EW EW EW EW EW EW EW EW EW EW EW $\begin{array}{llllllllllllllllllllllllllllllllllllllllll}24 & 25 & 26 & 27 & 28 & 29 & 30 & 31 & 32 & 33 & 34 & 35 & 36 & 37 & 38 & 39 & 40 & 41 & 42 & 43 & 44 & 45 & 46 & 47 & 48 & 49 & 50 & 51 & 52 & 1 & 2 & 3 & 4 & 5 & 6 & 7 & 8 & 9 & 10 & 11 & 12\end{array}$

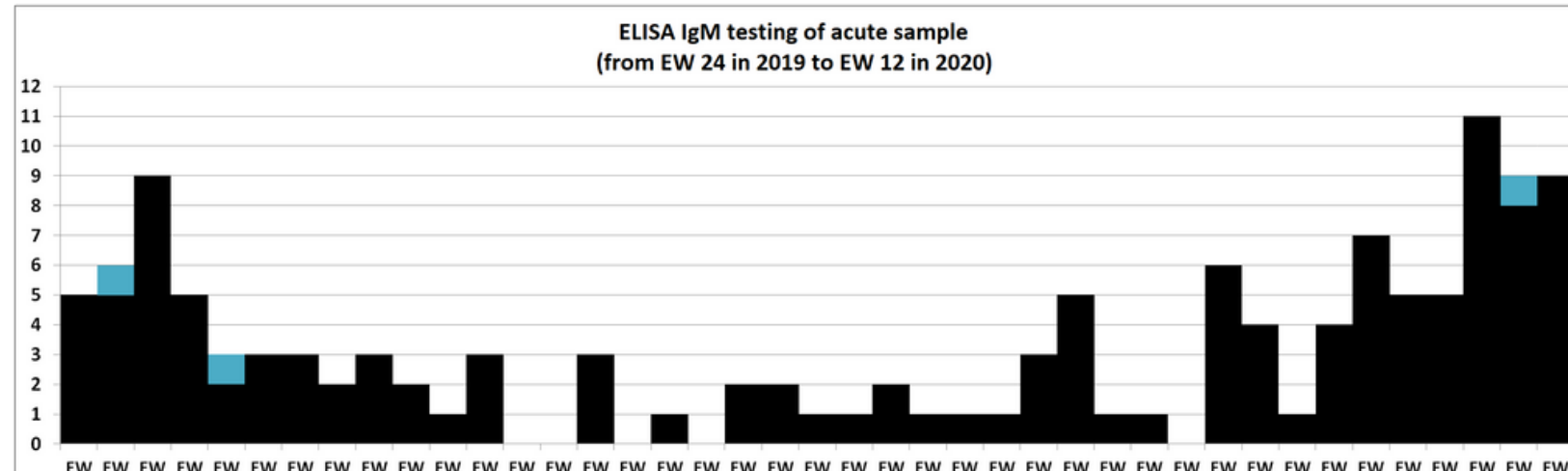

EW EW EW EW EW EW EW EW EW EW EW EW EW EW EW EW EW EW EW EW EW EW EW EW EW EW EW EW EW EW EW EW EW EW EW EW $\begin{array}{llllllllllllllllllllllllllllllllllllllllll}24 & 25 & 26 & 27 & 28 & 29 & 30 & 31 & 32 & 33 & 34 & 35 & 36 & 37 & 38 & 39 & 40 & 41 & 42 & 43 & 44 & 45 & 46 & 47 & 48 & 49 & 50 & 51 & 52 & 1 & 2 & 3 & 4 & 5 & 6 & 7 & 8 & 9 & 10 & 11 & 12\end{array}$

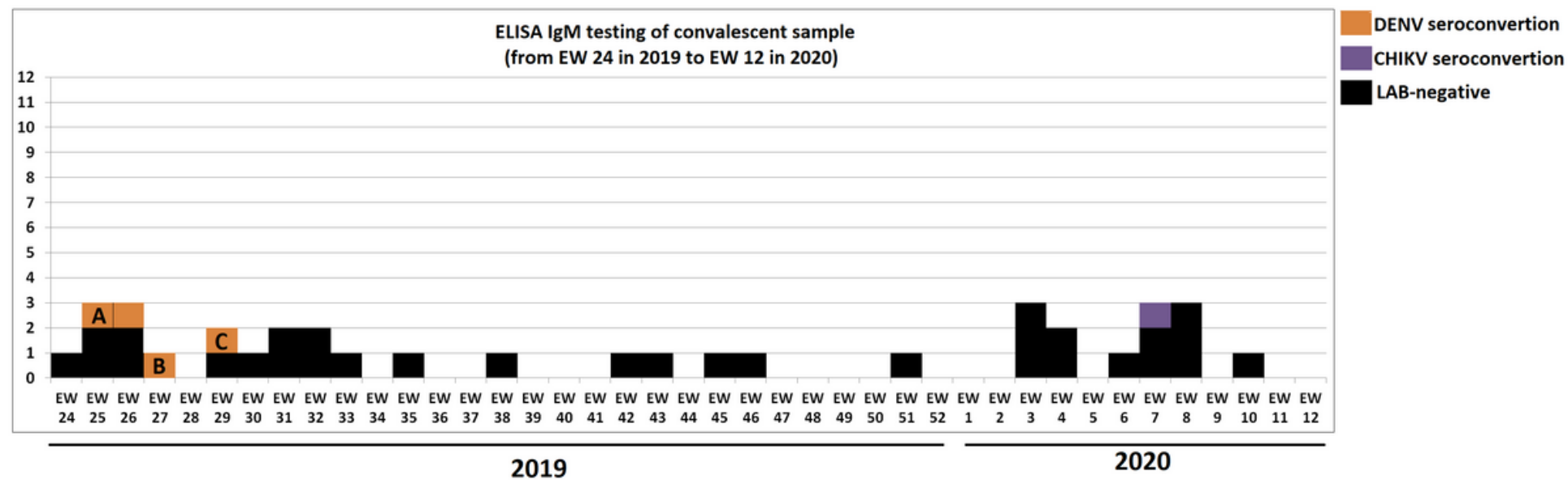

Figure 2

Epidemic curves for laboratory confirmations for LSS symptom onset data between EW 24 of 2019 and EW 12 of 2020, RT-PCR testing for acute samples (131); ELISA-IgM testing for acute samples (131); and ELISA-IgM testing for convalescent samples (36). A, B and C on the RT-PCR and Elisa-IgM curves for convalescent sample of the same individuals laboratory confirmed for acute DENV-2 infection. 


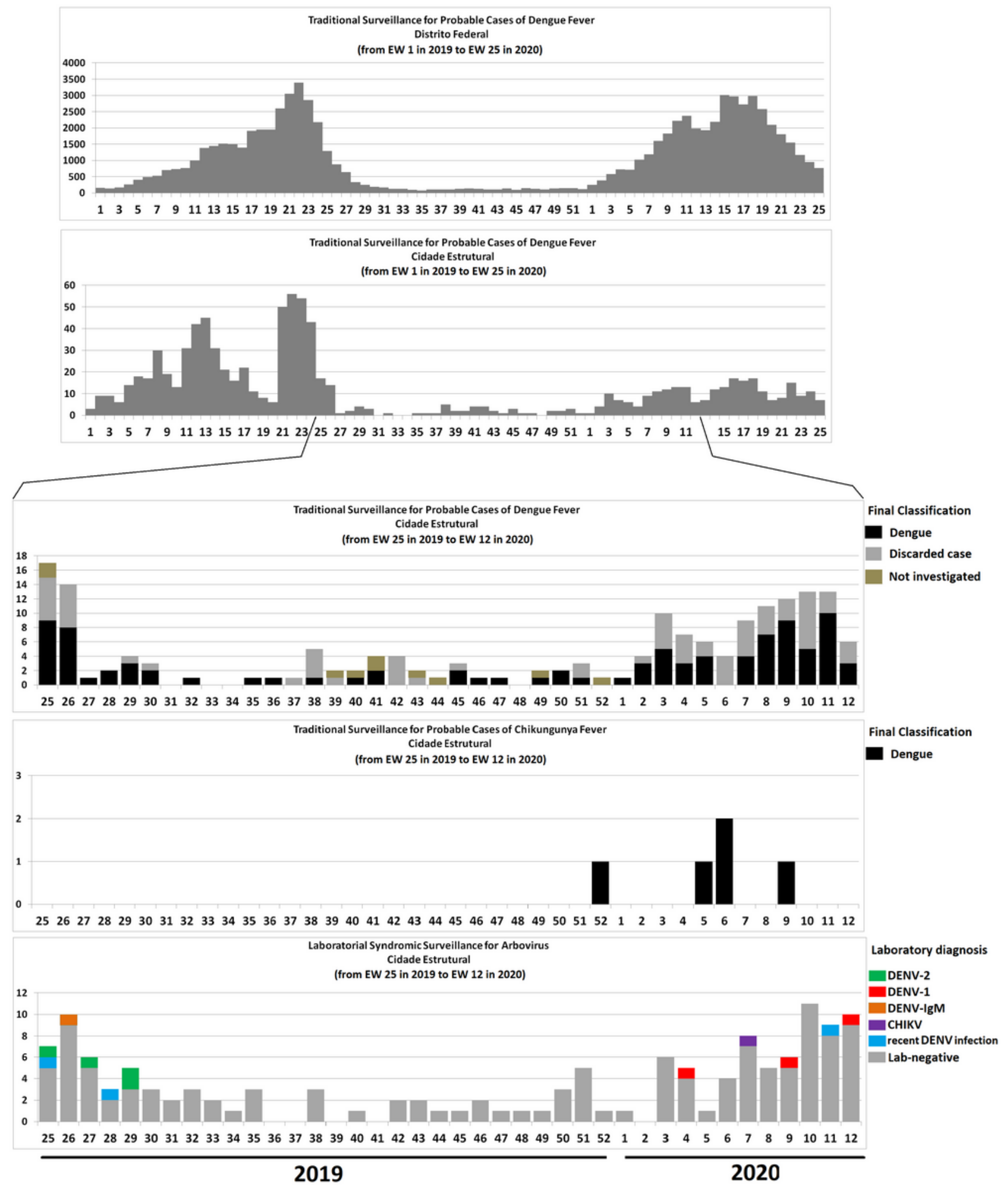

Figure 3

From top to bottom, epidemic curves of symptom onset for traditional surveillance of probable dengue cases in the Federal District between EW 1 of 2019 and EW 25 of 2020; traditional surveillance of probable dengue cases in Cidade Estrutural betwen EW 1 of 2019 and EW 25 of 2020; traditional surveillance of probable dengue cases and final classification for Cidade Estrutural between EW 25 of 2019 and EW 12 of 2020; traditional surveillance of probable Chikungunya cases and final classification 
for Cidade Estrutural between EW 25 of 2019 and EW 12 of 2020; and LSS results between EW 25 of 2019 and EW 12 of 2020.

\section{Supplementary Files}

This is a list of supplementary files associated with this preprint. Click to download.

- SupplementaryMaterial1.docx

- SupplementaryMaterial2.docx

- SupplementaryMaterial3.docx 